\title{
Statyba
}

\section{THE PROBLEMS OF LIMIT LOAD ANALYSIS AND OPTIMIZATION USING EQUILIBRIUM FINITE ELEMENTS}

\section{S. Kalanta}

To cite this article: S. Kalanta (1996) THE PROBLEMS OF LIMIT LOAD ANALYSIS AND OPTIMIZATION USING EQUILIBRIUM FINITE ELEMENTS, Statyba, 2:7, 13-23, DOI: 10.1080/13921525.1996.10531650

To link to this article: https://doi.org/10.1080/13921525.1996.10531650

进 Published online: 26 Jul 2012.

Submit your article to this journal ๘

Џ Article views: 48 


\section{РАВНОВЕСНЫЕ КОНЕЧНО-ЭЛЕМЕНТНЫЕ ПОСТАНОВКИ ЗАДАЧ РАСЧЕТА И ОПТИМИЗАЦИИ ПРЕДЕЛЬНОЙ НАГРУЗКИ}

\section{C. Каланта}

\section{1. Введение}

Обшие математические модели задач расчета и оптимизации предельной нагрузки для сплошного тела построены в [1,2]. Пластическое разрушение конструкщий обычно происходит изза образования поверхностей сосредоточенных пластических деформаций, в которых имеют место разрывы скоростей перемещений и диссипация энергии. Однако в равновесньх и двойственных математических моделях задач предельного равновесия тонкостенных конструкций [3-5 и др.] разрывы скоростей перемещений обычно не прогнозируются. Поэтому такие постановки задач не могут с достаточной точностью моделировать действительное поведение упругопластических пластинок и оболочек. Разрывы скоростей перемещений обычно учитываются только в кинематической постановке задачи расчета предельной нагрузки, построенной независимо от статической постановки $[6,7$ и др.] (Двойственные связи задач не соблюдаются). Проблема учета возможных разрывов перемещений в равновесных моделях рассматривалась нами в работах [8-10], посвященных задачам оптимизации жесткопцастического тела $[8,9]$ и задачам анализа напряженно-деформированного состояния (НДС) конструкций до пластического разруІІения [10]. Как продолжение исследований по совершенствованию метода конечных элементов применительно $\mathbf{k}$ упрутопластическим конструкциям в настоящей статье строятся двойственные равновесные математические модели задач расчета и оптимизации предельной нагрузки с учетом разрывов скоростей перемещений и скорости диссипации энергии в местах этих разрывов.

\section{2. Общие вариационные постановки задач}

Рассматривается жесткопластическое тело, геометрические параметры, условия опирания и функция $C(\mathbf{x})$ параметра текучести материала которого заданы. На поверхности $S_{f}$ тела распределена внешняя нагрузка $\mathbf{F}(\mathbf{x})$, a на поверхности $S_{u}$ заданы перемещения $\dot{\mathbf{u}}(\mathbf{x})=\mathbf{0}$.

Определяется НДС и оптимальное распределение или параметр нагрузки $F_{0}$, отвечаюцие предельному состоянию тела, возникающему изза исчерпания его прочности. Цискретная модель тела строится разделением его на $s$ конечньх элементов объема $V_{k}$, где $k=1,2, \ldots, s$ или $k \in \mathscr{K}$. НДС $k$-го элемента описывается вектор-функщиями напряжений $\sigma_{k}(\mathbf{x})$, скоростей перемещений $\dot{\mathbf{u}}_{k}(\mathbf{x})$ и деформаций $\dot{\varepsilon}_{k}(\mathbf{x})$. Функщии напряжений и скоростей перемещений, относящиеся к смежным конечным элементам, разделенным поверхностью $S_{t}$, обозначаются знаками “ + " и “-”. Пусть номера $t=1,2, \ldots, z$ поверхностей $S_{t}$ конечных элементов образукт множество $\mathscr{T}=\mathscr{T}_{v} \cup \mathscr{T}, \bigcup \mathscr{T}_{*}$, где $\mathscr{S}_{v}-$ множество номеров внутренних межэлементньх поверхностей; $\mathscr{T}_{f}$ множество номеров граничных, принадлежащих $S_{f}$, поверхностей элементов; オ̛ - множество номеров граничньх поверхностей $S_{u t}$. Пусть множество $\mathbb{T}_{f}=g \mathbb{T}_{\mathrm{V}} \cup T_{,}$. Математически модели задач строятся на основе статической теоремы предельного равновесия и метода преобразований Јагранжа. 


\section{1.Задача расчета параметра предельной нагрузки}

При заданном законе распределения нагрузки $\mathbf{F}(\mathbf{x})=F_{0} \eta(\mathbf{x})$ определяется параметр предельной нагрузки $F_{0}$ и НДС конструкции, отвечающие пластическому разрушению тела Здесь $\eta(\mathbf{x})$ - заданная функция распределения нагрузки

Мощность нагрузки

$$
\dot{W}=\int_{S_{f}} \dot{\mathbf{u}}^{T}(\mathbf{x}) \mathbf{F}(\mathbf{x}) d S=\sum_{t \in \mathscr{F}_{j}} F_{0} \int_{S_{f t}} \dot{\mathbf{u}}_{t}^{T}(\mathbf{x}) \eta_{t}(\mathbf{x}) d S_{t}
$$

На основе статической теоремы предельного равновесия [1] при фиксированных скоростях перемещений

$$
\sum_{t \in \mathscr{F}_{,}} \int_{S_{f t}} \dot{\mathbf{u}}_{t}^{T}(\mathbf{x}) \eta_{t}(\mathbf{x}) d S_{t}=1
$$

получается следующая математическая модель статической постановки задачи определения параметра предельной нагрузки

$$
F_{0} \rightarrow \mathbf{m a x}
$$

при условиях

$$
\begin{aligned}
& {[\mathscr{A}] \sigma_{k}(\mathbf{x})=\mathbf{0}, \quad \mathbf{f}\left(\sigma_{k}(\mathbf{x})\right) \leq \mathbf{f}_{0}\left(C_{k}(\mathbf{x})\right) \in V_{k}, \quad k \in \mathscr{K} ; } \\
& \mathbf{f}_{t}\left(\mathbf{p}_{t}^{-}(\mathbf{x})\right) \leq \mathbf{f}_{0 t}\left(C_{t}^{-}(\mathbf{x})\right) \in S_{t}^{-}, \quad t \in \mathscr{T} ; \\
& \mathbf{f}_{t}\left(\mathbf{p}_{t}^{+}(\mathbf{x})\right) \leq \mathbf{f}_{0 t}\left(C_{t}^{+}(\mathbf{x})\right) \in S_{t}^{+}, \quad t \in \mathscr{T} ; \\
& {\left[A_{t}\right] \sigma_{t}^{-}(\mathbf{x})+\mathbf{p}_{t}^{-}(\mathbf{x})=\mathbf{0} \in S_{t}^{-}, \quad t \in \mathscr{T} ; } \\
&- {\left[A_{t}\right] \sigma_{t}^{+}(\mathbf{x})+\mathbf{p}_{t}^{+}(\mathbf{x})=\mathbf{0} \in S_{t}^{+}, \quad t \in \mathscr{T} ; } \\
&- \mathbf{p}_{t}^{-}(\mathbf{x})-\mathbf{p}_{t}^{+}(\mathbf{x})+F_{0} \eta_{t}(\mathbf{x})=\mathbf{0} \in S_{t}, \quad t \in \mathscr{T}_{v} ; \\
&- \mathbf{p}_{t}^{+}(\mathbf{x})+F_{0} \eta_{t}(\mathbf{x})=\mathbf{0} \in S_{t}, t \in \mathscr{T}_{f} .
\end{aligned}
$$

Систему ограничений задачи, определяющую множество статически допустимых векторов напряжений, составляют уравнения равновесия и условия текучести внутри конечных элементов (первая строка) и на поверхностях элементов.

Методом преобразований Лагранжа задача (3) может быть преобразована в задачу стационарности:

$$
\mathscr{F}=\mathscr{F}_{0}+F_{0}\left\{1-\sum_{t \in \mathscr{O}, S_{t}} \int_{S_{t}} \dot{\mathbf{u}}_{t}^{T}(\mathbf{x}) \eta_{t}(\mathbf{x}) d S_{t}\right\} \rightarrow \text { stac },
$$

где

$$
\begin{aligned}
& \mathscr{F}_{t}=\sum_{t \in T_{\mathrm{S}}} \int_{S_{t}} \dot{\mathbf{u}}_{t}^{T}(\mathbf{x})\left\{\mathbf{p}_{t}^{-}(\mathbf{x})-\mathbf{p}_{t}^{+}(\mathbf{x})\right\} d S_{t}- \\
& -\sum_{t \in T_{j}} \int_{S_{i}} \dot{\mathbf{u}}_{t}^{T}(\mathbf{x}) \mathbf{p}_{t}^{+}(\mathbf{x}) d S_{t}-\sum_{k} \int_{V_{*}} \dot{\mathbf{u}}_{k}^{T}(\mathbf{x})[\propto \mathcal{l}] \sigma_{k}(\mathbf{x}) d V_{k}+ \\
& +\sum_{k} \int_{V_{k}} \dot{\lambda}_{k}^{T}(\mathbf{x})\left\{\mathbf{f}_{0}\left(C_{k}(\mathbf{x})\right)-\mathbf{f}\left(\sigma_{k}(\mathbf{x})\right)\right\} d V_{k}+ \\
& +\sum_{t \in \mathcal{T}} \int_{S_{t}^{-}}\left\{i_{t}^{-}(\mathbf{x})\right\}^{T}\left\{\mathbf{f}_{0 t}\left(C_{t}^{-}(\mathbf{x})\right)-\mathbf{f}_{t}\left(\mathbf{p}_{t}^{-}(\mathbf{x})\right)\right\} d S_{t}+ \\
& +\sum_{t \in O} \int_{S_{t}^{+}}\left\{\dot{\lambda}_{t}^{+}(\mathbf{x})\right\}^{T}\left\{\mathbf{f}_{0 t}\left(C_{t}^{+}(\mathbf{x})\right)-\mathbf{f}_{t}\left(\mathbf{p}_{t}^{+}(\mathbf{x})\right)\right\} d S_{t}- \\
& -\sum_{t \in \mathscr{T}_{v}} \int_{S}\left\{\dot{\mathbf{u}}_{t}^{-}(\mathbf{x})\right\}^{T}\left\{\left[A_{t}\right] \sigma_{t}^{-}(\mathbf{x})+\mathbf{p}_{t}^{-}(\mathbf{x})\right\} d S_{t}- \\
& -\sum_{t \in \mathscr{O}, S_{t}} \int_{S^{\prime}}\left\{\dot{\mathbf{u}}_{t}^{+}(\mathbf{x})\right\}^{T}\left\{-\left[A_{t}\right] \sigma_{t}^{+}(\mathbf{x})+\mathbf{p}_{t}^{+}(\mathbf{x})\right\} d S_{t} ; \\
& \dot{\lambda}_{k}(\mathbf{x}) \geq 0 \quad \in V_{k} ; \quad \dot{\lambda}_{t}^{-}(\mathbf{x}) \geq 0, \quad \dot{\lambda}_{t}^{+}(\mathbf{x}) \geq 0 \quad \in S_{t} .
\end{aligned}
$$

В случае статически допустимых напряжений два первых интеграла функционала Ғ выражают мощность нагрузки, а все остальные интегралы равны нулю. Применив к третьему интегралу формулу Гаусса-Остроградского, можно получить вторую форму смешанного функционала, которая для скоростей перемещений, удовлетворяющих геометрическим уравнениям, имеет энергетический смысл скорости диссипации энергии. Поэтому задачу стационарности (4) можно преобразовать в задачу минимизации скорости диссипации энергии, отвечающую кинематической теореме предельного равновесия

\section{2. Задача оптимизащии нагрузки}

При заданньх геометрических параметрах, условиях опирания и направлений нагрузки определяется оптимальное распределение нагрузки и НДС конструкции, отвечающие простому пластическому разрушению и заданному критерию оптимальности

$$
\max \varphi_{1}=\sum_{t \in \mathscr{T},} \int_{S_{y}} \mathbf{T}_{t}^{T}(\mathbf{x}) \mathbf{F}_{t}(\mathbf{x}) d \boldsymbol{S}_{t},
$$

где $\mathbf{T}_{t}(\mathbf{x})$ - векторная функщия весовых коэффициентов, означающих влияние (стоимость) нагрузки единичной пнтенсивности, 
распределенной на единичной площади. При единичных весовых функциях $\varphi_{1}$ представляет собой функцию суммарной нагрузки.

За условие огтимальности принимается условие постоянства мощности нагрузки

$$
\frac{\dot{\mathbf{u}}_{t}^{T}(\mathbf{x}) \mathbf{F}_{t}(\mathbf{x})}{\mathbf{T}_{t}^{T}(\mathbf{x}) \mathbf{F}_{t}(\mathbf{x})}=\text { const } \quad \text { или } \quad \dot{\mathbf{u}}_{t}^{T}(\mathbf{x}) \mathbf{F}_{t}(\mathbf{x})=c \mathbf{T}_{t}^{T}(\mathbf{x}) \mathbf{F}_{t}(\mathbf{x})
$$

для всех $t \in \mathscr{T}_{f}$, где $c$ - произвольная константа. Тогда функция $\varphi_{1}$ получает смысл мощности нагрузки и статическая постановка задачи огтимизации нагрузки выражается математической моделью:

$$
\sum_{t \in \mathscr{O}_{,}} \int_{S_{f t}} \mathbf{T}_{t}^{T}(\mathbf{x}) \mathbf{F}_{t}(\mathbf{x}) d S_{t} \rightarrow \max
$$

при условиях

$$
\begin{aligned}
& {[\mathscr{A}] \sigma_{k}(\mathbf{x})=\mathbf{0}, \mathbf{f}\left(\sigma_{k}(\mathbf{x})\right) \leq \mathbf{f}_{0}\left(C_{k}(\mathbf{x})\right) \in V_{k}, \quad k \in \mathscr{K} ; } \\
& \mathbf{f}_{t}\left(\mathbf{p}_{t}^{-}(\mathbf{x})\right) \leq \mathbf{f}_{0 t}\left(C_{t}^{-}(\mathbf{x})\right) \in S_{t}^{-}, \quad t \in \mathscr{F} ; \\
& \mathbf{f}_{t}\left(\mathbf{p}_{t}^{+}(\mathbf{x})\right) \leq \mathbf{f}_{0 t}\left(C_{t}^{+}(\mathbf{x})\right) \in S_{t}^{+}, \quad t \in \mathscr{F} ; \\
& {\left[A_{t}\right] \sigma_{t}^{-}(\mathbf{x})+\mathbf{p}_{t}^{-}(\mathbf{x})=\mathbf{0} \in S_{t}^{-}, \quad t \in \mathscr{F} ; } \\
&- {\left[A_{t}\right] \sigma_{t}^{+}(\mathbf{x})+\mathbf{p}_{t}^{+}(\mathbf{x})=\mathbf{0} \in S_{t}^{+}, \quad t \in \mathscr{F} ; } \\
&- \mathbf{p}_{t}^{-}(\mathbf{x})-\mathbf{p}_{t}^{+}(\mathbf{x})+\mathbf{F}_{t}(\mathbf{x})=\mathbf{0} \in S_{t}, \quad t \in \mathscr{F}_{\nu} ; \\
&- \mathbf{p}_{t}^{+}(\mathbf{x})+\mathbf{F}_{t}(\mathbf{x})=\mathbf{0}, \quad \mathbf{F}_{t}(\mathbf{x}) \geq \mathbf{0} \in S_{t}, t \in \mathscr{F}_{f} .
\end{aligned}
$$

Методом множителей Лагранжа она преобразуется в задачу стационарности функционала

$$
\mathscr{F}_{t}=\mathscr{F}_{0}+\sum_{t \in \mathscr{F}_{i} S_{t}} \int_{t} \mathbf{F}_{t}^{T}(\mathbf{x})\left\{\mathbf{T}_{t}(\mathbf{x})-\dot{\mathbf{u}}_{t}(\mathbf{x})+\dot{v}_{t}(\mathbf{x})\right\} d S_{t}
$$

при условиях (6). Принимая условия стационарности этого фунхционала по переменным $\sigma_{k}(\mathbf{x}), \mathbf{p}_{t}^{-}(\mathbf{x}), \mathbf{p}_{t}^{+}(\mathbf{x})$ и $\mathbf{F}_{t}(\mathbf{x})$ в качестве предварительных, получим кинематическую постановку задачи оптимизации нагрузки.

Обычно нагрузка действует лишь на некоторые из поверхностей $S_{t}$. Для поверхностей, $\mathrm{k}$ которым нагрузка не приложена, $-\mathbf{p}_{t}^{-}(\mathbf{x})=\mathbf{p}_{t}^{+}(\mathbf{x})=\mathbf{p}_{t}(\mathbf{x})$. С целью уменьшения числа неизвестных целесообразно для них применять такие условия сопряжения и текучести:

$$
\begin{aligned}
{\left[A_{t}\right] \sigma_{t}^{-}(\mathbf{x})-\mathbf{p}_{t}(\mathbf{x})=\mathbf{0} } & \in S_{t}^{-}, t \in \mathscr{T}_{v} ; \\
-\left[A_{t}\right] \sigma_{t}^{+}(\mathbf{x})+\mathbf{p}_{t}(\mathbf{x})=\mathbf{0} & \in S_{t}^{+}, t \in \mathscr{T}_{1} \\
\mathbf{f}_{t}\left(\mathbf{p}_{t}(\mathbf{x})\right) \leq \mathbf{f}_{0 t}\left(C_{t}(\mathbf{x})\right) & \in S_{t}, t \in \mathscr{T}
\end{aligned}
$$

Тогда статические граничные условия

$$
-\mathbf{p}_{t}(\mathbf{x})+\mathbf{F}_{t}(\mathbf{x})=\mathbf{0} \in S_{f t}, \quad t \in \mathscr{O T}_{f} .
$$

Соответственно преобразуется и функционал

При построении дискретных математических моделей задач вышеприведенные функциональные зависимости необходимо заменить дискретными аналогами. Для этого применяются равновесные конечные элементы.

\section{3. Равновесные дискретные здвисимости}

\section{1. Зависимости равновесного элемента}

Для построения дискретньх зависимостей конечного элемента и дискретной модели тела напряжения в каждом элементе аппроксимируются функциями

$$
\begin{aligned}
& \sigma_{k}(\mathbf{x})=\sum_{i=1}^{\xi}\left[H_{k i}(\mathbf{x})\right] \sigma_{k i}=\left[H_{k}(\mathbf{x})\right] \sigma_{k} \\
& \mathbf{p}_{t}(\mathbf{x})=\left[H_{t}(\mathbf{x})\right] \mathbf{p}_{t},
\end{aligned}
$$

при помощи которьх напряжения в любой точке элемента выражаются через векторы напряжений $\sigma_{k}, p_{t}$ узлов элемента. Ках и в работах [8,9], не требуется, чтобы функции (9) априорно удовлетворяли дифференциальным условиям равновесия в объеме элемента.

Вводится вектор обобщенных сил $\mathbf{P}_{k}$ и соответствующий ему вектор обобщенных перемещений $\dot{\mathbf{u}}_{k}$. Силы $\mathbf{P}_{k}$ и узловые напряжения $\sigma_{k}$. связаны уравнением равновесия элемента

$$
\mathbf{P}_{k}=\left[A_{k}\right] \mathbf{Q}_{k} \quad \text { или } \quad \mathbf{P}_{k}=\left[A_{\boldsymbol{\sigma} k}\right] \sigma_{k}+\left[A_{p k}\right] \mathbf{p}_{k} .
$$

В тех случаях, когда функции (9) тождественно не удовлетворяют дифференциальным условиям равновесия, вводятся дополнительные степени свободы и дополнительные уравнения равновесия элемента. Возможны два способа построения уравнений (10) - прямой и вариационный. Прямой способ заключается в прямом 
составлении уравнений равновесия по направлению выбранньх обобщенных сит и перемещений. При этом обобщенные силы и перемешения выбираются с таким расчетом, чтобы полученные таким образом уравнения равновесия дискретной модели конструкции гарантировали соблюдение условий равновесия во всех точках внутри и на границах элементов. По второму способу построения уравнения (10) получаются как условия стационарности дискретно описанного функционала (4) или (8) по переменным $\dot{\mathbf{u}}_{k}$ и $\dot{\mathbf{u}}_{t}$. Здесь необходимо дополнительно задать функции перемещений и пластических множителей и построить дискретное выражение функционала.

Дискретные условия текучести конечного элемента также могут быть получены прямым способом с применением классических методов коллокаций $[10,11]$ или вариационным методом как условия стационарности дискретно описанного функционала по переменным $\dot{\lambda}_{k}, \dot{\lambda}_{t}^{-}$и $\dot{\lambda}_{t}^{+}$. Они представляются неравенствами

$$
\begin{aligned}
& \mathbf{f}_{k}\left(\sigma_{k}\right) \leq \mathbf{f}_{0 k}\left(\mathbf{C}_{k}\right) ; \\
& \mathbf{f}_{t}\left(\overrightarrow{\mathbf{p}_{t}^{-}}\right) \leq \mathbf{f}_{0 t}\left(\mathbf{C}_{t}^{-}\right), \quad \mathbf{f}_{t}\left(\mathbf{p}_{t}^{+}\right) \leq \mathbf{f}_{0 t}\left(\mathbf{C}_{t}^{+}\right) .
\end{aligned}
$$

Здесь

$$
\begin{aligned}
& \mathbf{f}_{k}\left(\sigma_{k}\right)=\int_{V_{k}}\left[G_{k}(\mathbf{x})\right]^{T} \mathbf{f}\left(\sigma_{k}(\mathbf{x})\right) d V_{k}, \\
& \mathbf{f}_{0 k}\left(\mathbf{C}_{k}\right)=\int_{V_{k}}\left[G_{k}(\mathbf{x})\right]^{T} \mathbf{f}_{0}\left(C_{k}(\mathbf{x})\right) d V_{k}, \\
& \mathbf{f}_{t}\left(\mathbf{p}_{t}\right)=\int_{S_{k}}\left[G_{t}(\mathbf{x})\right]^{T} \mathbf{f}_{t}\left(\mathbf{p}_{t}(\mathbf{x})\right) d S_{t}, \\
& \mathbf{f}_{t}\left(\mathbf{C}_{t}\right)=\int_{S_{k}}\left[G_{t}(\mathbf{x})\right]^{T} \mathbf{f}_{0 t}\left(C_{t}(\mathbf{x})\right) d S_{t} .
\end{aligned}
$$

Матрицы $\left[G_{k}(\mathbf{x})\right],\left[G_{t}(\mathbf{x})\right]$ являются матрицами функций формы пластических множителей или матрицами весовых функщий соответствующих методов коллокаций. В работе [10] показано, что наилучшая аппроксимация условий текучести, пластических деформаций и скорости диссипации энергии получается с применением метода коллокаций Бубнова-Галеркина. Задавая функщии
$\mathbf{f}\left(\sigma_{k}(\mathbf{x})\right)=\sum_{i=1}^{\xi}\left[H_{f k i}(\mathbf{x})\right] \mathbf{f}_{k i}\left(\sigma_{k i}\right)=\left[H_{f k}(\mathbf{x})\right] \mathbf{f}_{k}\left(\sigma_{k}\right)$,

можно пгосроить упрощенные выражения интегра.льных ус.товий текучести [11].

Геометрические уравнения элемента строятся на основе принципа виртуальньх сил или вариационным методом из условия стационарности дискретно описанного функщионала по переменным $\sigma_{k}$ и $\mathbf{p}_{t}$. Они выражают совместность скоростей перемещений и пластических деформаций внутри элемента и на его поверхностях и имеют следующий вид:

$$
\begin{gathered}
{\left[\nabla \mathbf{f}_{k}\left(\sigma_{k}\right)\right]^{T} \dot{\lambda}_{k}-\left[A_{\boldsymbol{\sigma} k}\right]^{T} \dot{\mathbf{u}}_{k}=\mathbf{0},} \\
{\left[\nabla \mathbf{f}_{t}\left(\mathbf{p}_{t}\right)\right]^{T} \dot{\lambda}_{t}-\left[A_{p k}\right]^{T} \dot{\mathbf{u}}_{t}=\mathbf{0},}
\end{gathered}
$$

где $\left[\nabla \mathbf{f}_{k}\left(\sigma_{k}\right)\right],\left[\nabla \mathbf{f}_{t}\left(\mathbf{p}_{t}\right)\right]$ - матрицы градиентов условий текучести конечного элемента. Пластические множители $\dot{\lambda}_{k} \geq \mathbf{0}, \dot{\lambda}_{t} \geq \mathbf{0}$ и удовлетворяют условиям

$$
\dot{\lambda}_{k}^{T}\left\{\mathbf{f}_{0 k}\left(\mathbf{C}_{k}\right)-\mathbf{f}_{k}\left(\sigma_{k}\right)\right\}=\mathbf{0}, \quad \dot{\lambda}_{t}^{T}\left\{\mathbf{f}_{0 t}\left(\mathbf{C}_{t}\right)-\mathbf{f}_{t}\left(\mathbf{p}_{t}\right)\right\}=\mathbf{0}
$$

\section{2. Зависимости дискретной модели конструкцни}

Пусть внешняя нагрузка дискретной модели конструкщии описывается вектором $F$, a перемешения - вектором и. Уравнения (10) -(12) $k$-го элемента не связаны с уравнениями остальных элементов. Объединение свободньх конечных элементов в дискретную модель конструкщии осуществляется при помощи уравнений совместности перемешений

$$
\overline{\mathbf{u}}=[C] \dot{\mathbf{u}},
$$

которые определяют связь обобщенных перемещений конечных элементов и дискретной модели конструкщии.

Уравнения равновесия дискретной модели строятся на основе принцина возможных перемещений и получают вид:

$$
[C]^{T} \mathbf{P}=\mathbf{F} \quad \text { или } \quad\left[A_{\sigma}\right] \sigma+\left[A_{p}\right] \mathbf{p}=\mathbf{F},
$$


где

$$
\left[A_{\boldsymbol{\sigma}}\right]=[C]^{T}\left[\bar{A}_{\boldsymbol{\sigma}}\right], \quad\left[A_{p}\right]=[C]^{T}\left[\bar{A}_{p}\right]
$$

Здесь $\left[\bar{A}_{\sigma}\right],\left[\bar{A}_{p}\right]$ - квазидиагональные матрицы, диагональными блоками которых являются матрицы $\left[A_{\sigma k}\right],\left[A_{p k}\right]$.Составляющими векторов $\mathbf{P}, \sigma, \mathbf{p}$ являются соответственно векторы $\mathbf{P}_{k}, \sigma_{k}, \mathbf{p}_{k}$. С применением зависимостей (13), (15) геометрические уравнения свободных элементов преобразуются в глобальные геометрические уравнения дискретной модели.

Итак, НДС дискретной модели конструкции описываются следующими зависимостями:

$$
\begin{aligned}
& {\left[A_{\boldsymbol{\sigma}}\right] \sigma+\left[A_{p}\right] \mathbf{p}=\mathbf{F},} \\
& \mathbf{f}(\boldsymbol{\sigma}) \leq \mathbf{f}_{0}(\mathbf{C}), \quad \mathbf{f}_{p}(\mathbf{p}) \leq \mathbf{f}_{0 p}(\mathbf{C}) ; \\
& {[\nabla \mathbf{f}(\sigma)]^{T} \dot{\lambda}-\left[A_{\boldsymbol{\sigma}}\right]^{T} \dot{\mathbf{u}}=\mathbf{0}, \quad \quad \dot{\lambda} \geq \mathbf{0},} \\
& {\left[\nabla \mathbf{f}_{p}(\mathbf{p})\right]^{T} \dot{\lambda}_{p}-\left[A_{p}\right]^{T} \dot{\mathbf{u}}=\mathbf{0}, \quad \dot{\lambda}_{p} \geq \mathbf{0} ;} \\
& \dot{\lambda}^{T}\left\{\mathbf{f}_{0}(\mathbf{C})-\mathbf{f}(\sigma)\right\}=\mathbf{0}, \quad \dot{\lambda}_{p}^{T}\left\{\mathbf{f}_{0 p}(\mathbf{C})-\mathbf{f}_{p}(\mathbf{p})\right\}=\mathbf{0} .
\end{aligned}
$$

Две группы условий текучести и геометрических уравнений можно объединить:

$$
\tilde{\mathbf{f}}(\mathbf{Q}) \leq \tilde{\mathbf{f}}_{0}(\mathbf{C}), \quad[\nabla \tilde{\mathbf{f}}(\mathbf{Q})]^{T} \tilde{\dot{\lambda}}-[A]^{T} \dot{\mathbf{u}}=\mathbf{0},
$$

где $[A] \equiv\left[\left[A_{\boldsymbol{\sigma}}\right],\left[A_{p}\right]\right], \mathbf{Q} \equiv\{\sigma, \mathbf{p}\}^{T}, \tilde{\dot{\lambda}} \equiv\left\{\dot{\lambda}, \dot{\lambda}_{p}\right\}^{T}$, $\tilde{\mathbf{f}}(\mathbf{Q}) \equiv\{\mathbf{f}(\sigma), \mathbf{f}(\mathbf{p})\}^{T}$. Тогда, выбирая базисную матрицу $\left[A_{1}\right]^{T}$ и используя решение

$$
\dot{\mathbf{u}}=\left(\left[A_{1}\right]^{T}\right)^{-1}[\nabla \tilde{\mathbf{f}}(\mathbf{Q})]^{T} \dot{\lambda},
$$

геометрические уравнения можно преобразовать в уравнения совместности скоростей пластических деформаций

$$
[B(\mathbf{Q})] \dot{\boldsymbol{\lambda}}=\mathbf{0},
$$

где $[B(\mathbf{Q})]=\left[\nabla \tilde{\mathbf{f}}_{2}(\mathbf{Q})\right]^{T}-\left[A_{2}\right]^{T}\left(\left[A_{1}\right]^{T}\right)^{-1}\left[\nabla \tilde{\mathbf{f}}_{1}(\mathbf{Q})\right]^{T}$. Здесь $[\nabla \widetilde{\mathbf{f}}(\mathbf{Q})]^{T}-$ матрица градиентов условий текучести, соответствующая матрище $\left[A_{1}\right]^{T}$. Зависимость (19) позволяет исключить скорости перемешений из числа неизвестных задач.

\section{4. Дискретные математические модели}

Приведем двойственные пары дискретных математических моделей задач в статической и кинематической постановхах. Прежде всего с применением дискретных выражений уравнений равновесия и условий текучести (16) на основе общих математических моделей (3) и (7) получаем дискретные статические постановки задач. Далее методом множителей Лагранжа они преобразуются в кинематические постановки.

Задача расчета параметра нагрузки:

а) в статической постановке -

$$
F_{0} \rightarrow \max
$$

при условиях

$$
\begin{gathered}
\mathbf{f}(\sigma) \leq \mathbf{f}_{0}(\mathbf{C}), \quad \mathbf{f}_{p}(\mathbf{p}) \leq \mathbf{f}_{0 p}(\mathbf{C}), \\
-\left[A_{\boldsymbol{\sigma}}\right] \sigma-\left[A_{p}\right] \mathbf{p}+F_{0} \boldsymbol{\eta}=\mathbf{0}
\end{gathered}
$$

6) в кинематической постановке -

$$
\begin{gathered}
\dot{\lambda}^{T}[\nabla \mathbf{f}(\sigma)] \sigma+\dot{\lambda}_{p}^{T}\left[\nabla \mathbf{f}_{p}(\mathbf{p})\right] \mathbf{p}+\dot{\lambda}^{T}\left\{\mathbf{f}_{0}(\mathbf{C})-\mathbf{f}(\sigma)\right\}+ \\
+\dot{\lambda}_{p}^{T}\left\{\mathbf{f}_{0 p}(\mathbf{C})-\mathbf{f}_{p}(\mathbf{p})\right\} \rightarrow \min
\end{gathered}
$$

при условиях

$$
\begin{aligned}
& {[\nabla \mathbf{f}(\sigma)]^{T} \dot{\lambda}-\left[A_{\boldsymbol{\sigma}}\right]^{T} \dot{\mathbf{u}}=\mathbf{0},} \\
& {\left[\nabla \mathbf{f}_{p}(\mathbf{p})\right]^{T} \dot{\lambda}_{p}-\left[A_{p}\right]^{T} \dot{\mathbf{u}}=\mathbf{0},} \\
& \eta^{T} \dot{\mathbf{u}}=1, \quad \dot{\lambda} \geq \mathbf{0}, \quad \dot{\lambda}_{p} \geq \mathbf{0} .
\end{aligned}
$$

Первый и второй члены функционала задачи (22) выражают скорость диссипации энергии в объеме и на поверхностях конечных элементов, а остальные члены для оптимального решения равны нулю.

Задача оптимизации нагрузки. Дискретные математические модели этой задачи строятся с применением дискретньх зависимостей (16)-(18) и

$$
\dot{W}=\sum_{t} \int_{S_{f t}} \mathbf{T}_{t}^{T}(\mathbf{x})\left[H_{f}(\mathbf{x})\right] \mathbf{F}_{t}(\mathbf{x}) d S_{t}=\sum_{t} \mathbf{T}_{t}^{T} \mathbf{F}_{t}=\mathbf{T}^{T} \mathbf{F} .
$$

В общем случае составляющие вектора $\mathbf{F}$ могут быть линейно зависимы, поэтому вводится вектор оाтимизируемых параметров $\mathbf{F}_{0}$ и устанавливается связь 


$$
\mathbf{F}=[\eta] \mathbf{F}_{0} .
$$

Тогда мощность нагрузки

$$
\dot{W}=\mathbf{T}^{T}[\eta] \mathbf{F}_{0}=\mathbf{T}_{0}^{T} \mathbf{F}_{0}, \quad \text { где } \quad \mathbf{T}_{0}=[\eta]^{T} \mathbf{T} .
$$

Получается следующая двойственная пара математических моделей задачи огтимизации нагрузки:

а) в статической постановке -

при условиях

$$
\mathbf{T}_{0}^{T} \mathbf{F}_{0} \rightarrow \mathbf{m a x}
$$

$$
\begin{aligned}
& \mathbf{f}(\sigma) \leq \mathbf{f}_{0}(\mathbf{C}), \quad \mathbf{f}_{p}(\mathbf{p}) \leq \mathbf{f}_{0 p}(\mathbf{C}), \\
& -\left[A_{\sigma}\right] \sigma-\left[A_{p}\right] \mathbf{p}+[\eta] \mathbf{F}_{0}=\mathbf{0}, \quad-\mathbf{F}_{0} \leq \mathbf{0} ;
\end{aligned}
$$

б) в кинематической постановке -

$$
\begin{gathered}
\dot{\lambda}^{T}[\nabla \mathbf{f}(\sigma)] \sigma+\dot{\lambda}_{p}^{T}\left[\nabla \mathbf{f}_{p}(\mathbf{p})\right] \mathbf{p}+\dot{\lambda}^{T}\left\{\mathbf{f}_{0}(\mathbf{C})-\mathbf{f}(\sigma)\right\}+ \\
+\dot{\lambda}_{p}^{T}\left\{\mathbf{f}_{0 p}(\mathbf{C})-\mathbf{f}_{p}(\mathbf{p})\right\} \rightarrow \min
\end{gathered}
$$

при условиях

$$
\begin{aligned}
& {[\nabla \mathbf{f}(\boldsymbol{\sigma})]^{T} \dot{\lambda}-\left[A_{\mathbf{\sigma}}\right]^{T} \dot{\mathbf{u}}=\mathbf{0},} \\
& {\left[\nabla \mathbf{f}_{p}(\mathbf{p})\right]^{T} \dot{\lambda}_{P}-\left[A_{p}\right]^{T} \dot{\mathbf{u}}=\mathbf{0},} \\
& {[\eta]^{T} \dot{\mathbf{u}} \geq \mathbf{T}_{0}, \quad \dot{\lambda} \geq \mathbf{0}, \quad \dot{\lambda}_{p} \geq \mathbf{0} .}
\end{aligned}
$$

Двойственные пары задач математического программирования (21), (22) и (23), (24) отвечают статической и кинематической теоремам предельного равновесия. Они построены с применением равновесных конечных элементов и позволяют определить нижние оценки предельной нагрузки и параметров напряженнодеформированного состояния. В тех случаях, когда вектор $\mathbf{F}_{0}$ содержит лишь один параметр $\left(\mathbf{F}_{0} \equiv F_{0}\right)$, задачи (23) и (24) превращаются в пару задач расчета параметра предельной нагрузки, т.е. эквивалентны задачам (21), (22).

Если, кроме варьируемой, действует и постоянная нагрузка и (или) другие воздействия (осадка опор, начальные деформации), то математические модели задачи оптимизации нагрузки преобразуются в следующие:

а) статическая постановка -

$$
\mathbf{T}_{0}^{T} \mathbf{F}_{0} \rightarrow \mathbf{m a x}
$$

при условиях

$$
\begin{aligned}
\mathbf{f}(\sigma) \leq \mathbf{f}_{0}(\mathbf{C}), \quad \mathbf{f}_{p}(\mathbf{p}) \leq \mathbf{f}_{0 p}(\mathbf{C}), \\
-\left[A_{\boldsymbol{\sigma}}\right] \sigma-\left[A_{p}\right] \mathbf{p}+[\eta] \mathbf{F}_{0}=-\mathbf{F}_{1}, \quad-\mathbf{F}_{0} \leq \mathbf{0} ;
\end{aligned}
$$

б) кинематическая постановка -

$$
\begin{aligned}
& \dot{\lambda}^{T}[\nabla \mathbf{f}(\sigma)] \sigma+\dot{\lambda}_{p}^{T}\left[\nabla \mathbf{f}_{p}(\mathbf{p})\right] \mathbf{p}-\mathbf{F}_{1}^{T} \dot{\mathbf{u}}+ \\
+ & \dot{\lambda}^{T}\left\{\mathbf{f}_{0}(\mathbf{C})-\mathbf{f}(\sigma)\right\}+\dot{\lambda}_{p}^{T}\left\{\mathbf{f}_{0 p}(\mathbf{C})-\mathbf{f}_{p}(\mathbf{p})\right\} \rightarrow \mathbf{m i n}
\end{aligned}
$$

при условиях (17) и

$$
[\eta]^{T} \dot{\mathbf{u}} \geq \mathbf{T}_{0}
$$

Здесь $\mathbf{F}_{1}$ - вектор постоянной нагрузки. При наличии начальных деформаций или осадки опор $\mathbf{F}_{1}$ представляет собой вектор нагрузки, эквивалентный этим воздействиям. Он определяется упругим расчетом конструкщии [12]. Постоянные воздействия также будут учтены, если функции текучести в математических моделях (23), (24) заменим на функщии $\mathbf{f}\left(\sigma+\sigma_{e}\right)$ и $\quad \mathbf{f}\left(\mathbf{p}+\mathbf{p}_{e}\right), \quad$ где $\sigma_{e}, \mathbf{p}_{e}-$ векторы напряжений упругого расчета от постоянных воздействий. Тогда действительные напряжения $\sigma^{*}=\sigma+\sigma_{\epsilon}$ и $\mathbf{p}^{*}=\mathbf{p}+\mathbf{p}_{\epsilon}$.

модифицированные математические модеи. Представленные кинематические постановки задач содержат много неизвестных. Однако их число можно уменьшить. Используя решение (19), можно из числа неизвестних исключить скорости перемещений. Тогда, например, математическая модель (24) преобразуется $\mathbf{K}$ следующему виду:

$$
\tilde{\dot{\lambda}}^{T}[\nabla \tilde{\mathbf{f}}(\mathbf{Q})] \mathbf{Q}+\tilde{\dot{\lambda}}^{T}\left\{\tilde{\mathbf{f}}_{0}(\mathbf{C})-\tilde{\mathbf{f}}(\mathbf{Q})\right\} \rightarrow \min
$$

при условиях

$$
\begin{aligned}
& {[B(\mathbf{Q})] \tilde{\dot{\lambda}}=\mathbf{0}, \quad \tilde{\dot{\lambda}} \geq \mathbf{0},} \\
& {[\eta]^{T}\left(\left[A_{1}\right]^{T}\right)^{-1}[\nabla \tilde{\mathbf{q}}(\mathbf{Q})]^{T} \tilde{\dot{\lambda}} \geq \mathbf{T}_{0} .}
\end{aligned}
$$

Заменив ограничение мощности нагрузки уравнением

$$
\eta^{T}\left(\left[A_{1}\right]^{T}\right)^{-1}[\nabla \widetilde{\mathbf{q}}(\mathbf{Q})]^{T} \ddot{\dot{\lambda}}=1,
$$


получим аналогичную модель задачи расчета параметра предельной нагрузки.

Есть и другой способ исключения скоростей перемешений и сокращения общего числа неизвестньх. Используя решение

$$
\mathbf{Q}=\left[B_{1}\right]\left\{\mathbf{F}_{1}+[\boldsymbol{\eta}] \mathbf{F}_{0}\right\}+\left[B_{2}\right] \mathbf{Q}_{2}
$$

уравнений равновесия, можно из статической постановки задач исключить базисные напряжения и уравнения равновесия. Получается задача

при условиях

$$
\mathbf{T}_{0}^{T} \mathbf{F}_{0} \rightarrow \max
$$

$$
\tilde{\mathbf{f}}\left(\mathbf{Q}_{2}, \mathbf{F}_{0}, \mathbf{F}_{1}\right) \leq \tilde{\mathbf{6}}(\mathbf{C}), \quad-\mathbf{F}_{0} \leq \mathbf{0} .
$$

Здесь $\left[B_{1}\right],\left[B_{2}\right]$ - матрицы частного и общего решений уравнений равновесия [12]. Тогда скорости перемещений в двойственной кинематической постановке

$\tilde{\dot{\lambda}}^{T}\left[\frac{\partial \tilde{\mathbf{f}}\left(\mathbf{Q}_{2}, \mathbf{F}_{0}, \mathbf{F}_{1}\right)}{\partial \mathbf{F}_{0}}\right] \mathbf{F}_{0}+\tilde{\dot{\lambda}}^{T}\left\{\tilde{\mathbf{6}}-\tilde{\mathbf{T}}\left(\mathbf{Q}_{2}, \mathbf{F}_{0}, \mathbf{F}_{1}\right)\right\} \rightarrow$ min

при условиях

$$
\begin{aligned}
& {\left[\frac{\partial \tilde{\mathbf{f}}\left(\mathbf{Q}_{2}, \mathbf{F}_{0}, \mathbf{F}_{1}\right)}{\partial \mathbf{Q}_{2}}\right]^{T} \tilde{\dot{\lambda}}=\mathbf{0}, \quad\left[\frac{\partial \tilde{\mathbf{f}}\left(\mathbf{Q}_{2}, \mathbf{F}_{0}, \mathbf{F}_{1}\right)}{\partial \mathbf{F}_{0}}\right]^{T} \tilde{\dot{\lambda}} \geq \mathbf{T}_{0},} \\
& \tilde{\dot{\lambda}} \geq \mathbf{0}
\end{aligned}
$$

отсутствуют. Неизвестными в этих задачах являются лишь линейно независимые напряжения $\mathbf{Q}_{2}$, вектор нагрузки $\mathbf{F}_{0}$ и пластические множители $\tilde{\dot{\lambda}}$. Сопоставляя ограничения задач (27) и (29), приходим к выводу, что ограничения задачи (29) представляют собой уравнения совместности скоростей пластических деформаций и ограничения мощности нагрузки. Таким образом, уравнения совместности деформаций могут быть построены в качестве условий стационарности функщионала Лагранжа

$$
\mathscr{F}_{2}=\mathbf{T}_{0}^{T} \mathbf{F}_{0}+\tilde{\dot{\lambda}}^{T}\left\{\tilde{\mathbf{f}_{0}}-\tilde{\mathbf{f}}\left(\mathbf{Q}_{2}, \mathbf{F}_{0}, \mathbf{F}_{1}\right)\right\}+\dot{v}^{T} \mathbf{F}_{0}
$$

по переменным $\mathbf{Q}_{2}$. Условие стационарности этого функционала по $\mathbf{F}_{0}$ дает ограничение мощности нагрузки. а задачи (28) и (29) преобразуются К двойственной пгаре задач:

а) статическая постановка -

при условиях

$$
\mathbf{T}_{0}^{T} \mathbf{F}_{0} \rightarrow \max
$$

$$
[\widetilde{\Phi}]\left(\left[B_{1}\right]\left\{\mathbf{F}_{1}+[\eta] \mathbf{F}_{0}\right\}+\left[B_{2}\right] \mathbf{Q}_{2}\right) \leq \widetilde{\mathbf{C}}, \quad-\mathbf{F}_{0} \leq \mathbf{0} ;
$$

б) кинематическая постановка -

$$
\widetilde{\mathbf{C}}^{T} \tilde{\dot{\lambda}}-\mathbf{F}_{1}^{T}\left[B_{1}\right]^{T}[\widetilde{\boldsymbol{\Phi}}]^{T} \tilde{\dot{\lambda}} \rightarrow \min
$$

при условия

$$
\left[B_{2}\right]^{T}[\tilde{\boldsymbol{\Phi}}]^{T} \tilde{\dot{\lambda}}=\mathbf{0}, \quad[\eta]^{T}\left[B_{1}\right]^{T}[\tilde{\Phi}]^{T} \tilde{\dot{\lambda}} \geq \mathbf{T}_{0}, \quad \tilde{\dot{\lambda}} \geq \mathbf{0} .
$$

Неизвестными в задаче (32) являются лишь пластические множители - скорости пластических деформаций.

\section{5. Численный андлиз}

Численными исследованиями илюстрируется не только применение равновесньх конечньх элементов в задачах предельного равновесия, но и точность результатов расчета в зависимости от способа дискретизации условий решение, необходимое для оценки точности численньх результатов, в качестве иллюстративного примера выбирается одноэтажная двухпролетная рама, находяшаяся под действием равномерно распределенной нагрузки интенсивностью $p=F_{0}$ и сосредоточенной силы $F=3 F_{0}$ (рис. 1). Несуцая способность сечений текучести. Чтобы опредетить аналитическое 
стержней задана предельным моментом $M_{0}$. Требуется определить параметр предельной нагрузки $F_{0}$ и распределение изгибающих моментов, соответствуюшие пластическому разрушению рамы и разным способам дискретизации условий текучести.

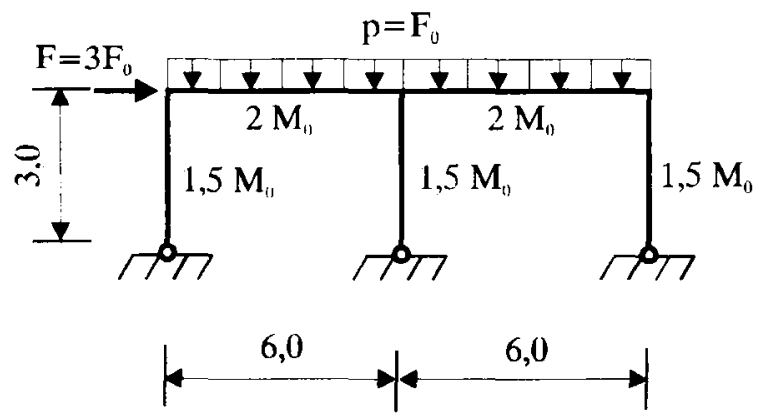

Рис. 1. Расчетная схема рамы

Fig. 1. Computing scheme of frame

a)

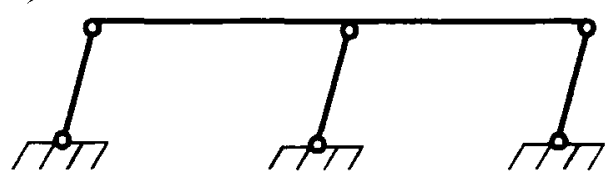

$\mathrm{F}_{0}=0,5 \mathrm{M}_{0}$

b)
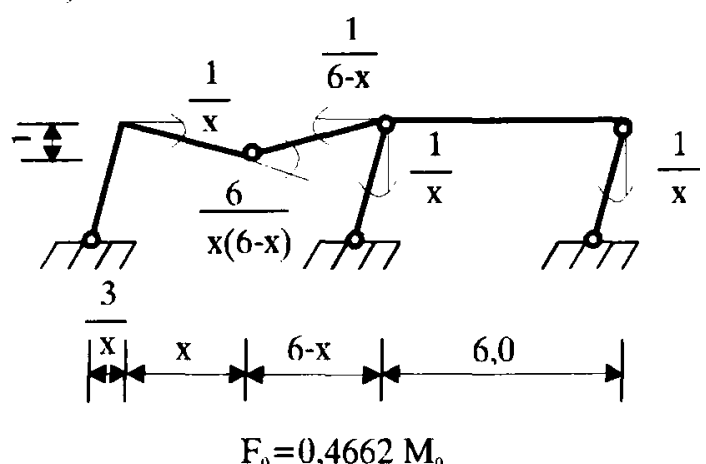

Pис. 2. Механизмы разрушения: $a$ ) бокового смещения; b) комбинированный

Fig. 2. Mechanisms of failure: a) side's overhanging, b) combinative

Аналитическое решение задачи определяется кинематическим методом предельного равновесия, основанным на исследовании кинематически возможных механизмов разрушения (применение статического метода связано с неоднократным решением системы уравнений равновесия). Ция каждого исследуемого механизма составляется уравнение равенства работ внешних и внутренних сил и определяется функщия параметра предельной нагрузки $F_{0}=F_{0}\left(x, M_{0}\right)$ и значение этого параметра. Если один пластический шарнир находится в ригеле рамы, то предварительно из условия экстремапьности

$$
d F_{0}\left(x, \Lambda I_{0}\right) / d x=0
$$

определяется местонахождение этого шшарнира, r.e. координата $\boldsymbol{x}$.

Возможные механизмы разрушения рассматриваемой рамы можно разделить на основные и комбинированные. $\mathrm{K}$ основным принадлежат механизм бокового смещения (рис. 2, а) и два балочных механизма разрушения риғелей. Комбинированные механизмы строятся "сложением" основных механизмов. Таким образом необходимо исследовать пять механизмов разрушения. Проиллюстрируем определение значения параметра $F_{0}$ лишь для одного комбинированного механизма (рис, 2,б).

Уравнение равенства работ внешних и внутренних сил

$$
\frac{9 F_{0}}{x}+3 F_{0}=\frac{12 M I_{0}}{x(6-x)}+\frac{2 M_{0}}{6-x}+\frac{3 M_{0}}{x} .
$$

Отсюда

$$
F_{0}=\frac{M_{0}(30-x)}{3\left(18+3 x-x^{2}\right)} .
$$

Условие экстремальности параметра $F_{0}$

$$
d F_{0} / d x=x^{2}-60 x+108=0,
$$

откуда координата пластического шарнира $x=1,8575 m$. С учетом этой координаты по.тучается значение $F_{0}=0.4662 M_{0}$. Параметры $F_{0}$. соответствуюшие остальным механизмам, превышают это значение. Поэтому действительными являются параметр преде.тьной нагрузки $F_{0}=0,4662 \mu_{0}$ и соответствуюший ему комби-

нированный механизм разрушения, показанный на ргіс. 2,6 .

Численный анализ рамы проводиля с применением равновесных конечных элементов первого и второго порядка и разньх условий текучести. Для дискретного описания колонн 
Табл. 1. Значения параметра предельной нагрузки $F_{0}$ и их погрешности $\mathcal{F}_{0}$

Table 1. Values of limit load parameter $F_{0}$ and computation errors $\Delta F_{0}$

\begin{tabular}{|c|c|c|c|c|c|c|c|c|c|}
\hline \multirow{3}{*}{$\begin{array}{c}\text { Порялок } \\
\text { конечного } \\
\text { элемента } \\
\end{array}$} & \multirow{3}{*}{$\begin{array}{c}\text { Условия } \\
\text { текучести }\end{array}$} & \multicolumn{8}{|c|}{ Число конечных элементов в ригеле } \\
\hline & & \multicolumn{2}{|c|}{1} & \multicolumn{2}{|c|}{2} & \multicolumn{2}{|c|}{3} & \multicolumn{2}{|c|}{4} \\
\hline & & $F_{0} / M_{0}$ & $\Delta F_{0}$ & $F_{0} / M_{0}$ & $\Delta F_{0}$ & $F_{0} / M_{0}$ & $\Delta F_{0}$ & $F_{0} / M_{0}$ & $\Delta F_{0}$ \\
\hline Первый & Точечные и др. & 0.5 & 7.25 & 0.5 & 7.25 & 0.4667 & 0.10 & 0.4691 & 0.63 \\
\hline \multirow{3}{*}{ Второй } & Точечные & 0.5 & 7.25 & 0.4691 & 0.63 & 0.4667 & 0.10 & 0.4691 & 0.63 \\
\hline & Интегр. точечные & 0.4630 & -0.69 & 0.4550 & -2.40 & 0.4575 & -1.86 & 0.4640 & -0.47 \\
\hline & Элементные & 0.5 & 7.25 & 0.4872 & 4.50 & 0.4667 & 0.10 & 0.4691 & 0.63 \\
\hline
\end{tabular}

использованы элементы первого порядка, а ригелей - элементы первого и второго порядка с соответственно линейным и параболическим распределением изгибающих моментов. В табл.1 приведены значения параметра предельной нагрузки $F_{0}$ (до множителя $M_{0}$ ) и процентная погрепность этих значений $\Delta F_{0}$ (отклонение от аналитического решения $F_{0}=0,4662 M_{0}$ ), полученные с применением точечных и интегральных условий текучести. Максимальное процентное нарушение условия прочности в опасном сечении находится в прямой пропорциональности от погрешности $\Delta F_{0}$ и определяется выражением $\Delta f=2,346 \Delta F_{0}$. Поэтому статически допустимые в глобальном смысле результаты расчета (изгибающие моменты удовлетворяют условиям равновесия и текучести во всех сечениях) получены лишь с ирименением интегральных точечных условий текучести. На рис. 3 иллюстрируется конверген-

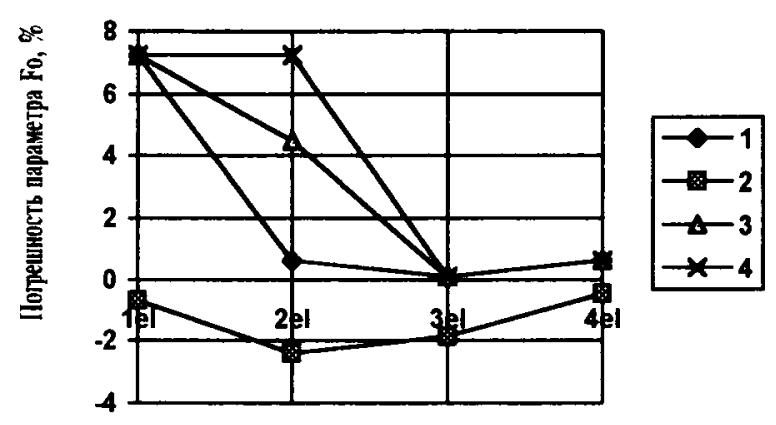

Рис. 3. Илгострация сходимости параметра $F_{0}$ для разных условий текучести

Fig. 3. Illustration of convergence of parameter $F_{0}$ for different yield conditions ция результатов расчета с применением разньх условий текучести: 1 - точечных, 2 - интегральных точечных, 3 - конечноэлементных, 4 для элементов первого порядка.

\section{6. Заключение}

На основе известньх теорем предельного равновесия [1,2] построены общие математические модели задач расчета и огтимизации предельной нагрузки, учитьваюшие возможные разрывы скоростей перемещений і скорость диссипации энергии не только в объеме, но и на поверхностях пластического разрушения конструкций. $\mathrm{C}$ применением равновесных конечных элементов и метода множителей Лагранжа построены дискретные выражения основньх зависимостей и дискретные двойственные математические модели статической и кинематической постановок задач. Показано, что применение уравнений совместности скоростей пластических деформаций позволяет формулировать задачи с менышим числом неизвестных. На примере простой двухпролетной рамы проведен качественный анализ разньх сгержневых элементов и условий текучести. Он подтверждает следующие теоретически прогнозируемые выводы:

1. Наиболее точные и стабильные результаты расщета, в наименышей степени зависяшие от густоты расчетной сетки, получаются с применением интегральных точечных условий текучести. 
2. Точность результатов расчета при точечных и конечноэлементных условиях текучести зависит не только от густоты, но и от успешного выбора расчетной сетки и не всегда улучшается при ее сгущении. Однако вероятность удачного выбора при сгушении расчетной сетки увеличивается.

3. Наиболее удачной является такая конечноэлементная сетка, узлы которой максимально приближены к местам пластического разрушения конструкщии.

4. В случае удачно выбранной расчетной сетки точность результатов расчета при точечних условиях текучести может даже превышать точность результатов, полученных с применением интегральньх точечных условий текучести.

\section{Литература}

1. А.А. Чирас. Теория оптимизации в предельном анализе твердого деформируемого тела. Вильнюс: Минтис, 1971. 123 с.

2. А.А. Чирас. Математические модели анализа и оптимизации упругопластических систем. Вильнюс: Мокслас, 1982. 112 с.

3. T. Belytschko, M. Velebit. Finite element method for elastic plastic plates//J. Eng. Mech. Div. Proc. Amer. Soc. Civ. Eng., 1972, 98, № 1, p. 227-242.

4. G. Maier, R.A. Zavelani, D. Bennedetti. A finite element approach to optimal design of plastic structures in plane stress//Int. Numer. Meth. Eng., 1972, 4, № 4, p. 455-473.

5. Ю.Ю. Аткочюнас. Расчет упругопластических систем при повторных нагружениях. Вильнюс: Изд-во науки и энциклопедий, 1994. $150 \mathrm{c}$.

6. A. Biron, G. Charleux. Limit analysis of axisymmetric pressure vessel intrsections of arbitrary shape//Int. J. Mech. Sci., 1972, 14, № 1, p. 25-41.

7. S. Turgeman, J. Pastor. Limit analysis: a linear formulation of the kinematic approach for axisymmetric mechanics problems//J. Int. Numer. and Anal. Meth. Geomech., 1982, 6, № 1, p. 109-128.

8. С.А. Каланта. Метод конечных элементов в задачах расчета и оптимизации жесткопластических систем при простом нагружении: Отчет о научно-исследовательской работе "Разработать метод конечных элементов применительно к задачам предельного равновесия". ГР № 0181100871, Инв. № 0206.0028660, Вильнюс, 1983. $71 \mathrm{c}$

9. С.А. Каланта. Двойственные математические модели задач оптимизации идеатьо пластичных систем конечных элементов// Приклацная механика и оптимизация (Литовскнй механический сборник, № 27), Вильнюс, 1985 , с. $40-48$.

10. С. Каланта. Двойственные математические модели задач анализа упругопластических конструкций с разрывными полями перемешений// Statyba (Строительство), № 3(3), Вильнюс: Техника, 1995, с. 5-29.

11. S. Kalanta. Takumo sąlygos baigtiniu elementu modeliams // Proceedings of 4th Int. Conf. "Modern building materials, structures and techniques", vol. III. Vilnius: Technika, 1995, p. 260-265.

12. С. Каланта. Равновесные конечные элементы в расчетах упругих конструкций//Statyba (Строительство), № 1(1), Вильнюс: Техника, 1995, с $25-47$.

Iteikta 19960903

\section{RIBINĖS APKROVOS ANALIZÉS IR OPTIMIZACLJOS UŽDAVINIU FORMULUOTÉS, PANAUDOJANT PUSIAUSVIRUOSIUS BAIGTINIUS ELEMENTUS}

\section{S. Kalanta}

\section{S a n t ra u a}

Sudaromi konstrukciju ribinès apkrovos ir optimizacijos uždaviniu dualūs diskretiniai matematiniai modeliai statine ir kinematine formuluote, panaudojant pusiausviruosius baigtinius elementus. Juose jvertinami galimi poslinkių greičiu trūkiai ir energijos disipacijos greitis ne tik kūno tūryje, bet ir plastiniuose paviršiuose tarp baigtinių elementų.

Remiantis statine teorema apie ribinę apkrovą [1, 2], pirmiausia sudaryti uždavinių bendri matematiniai modeliai (3) ir (7). Sių modelių diskretizacijai naudojami pusiausvirieji baigtiniai elementai ir itempimy aproksimavimo funkcijos (9). Optimalumo kriterijumi pasirinkta išorinès apkrovos galingumo pastovumo sąlyga. Sudarytos pusiausvyros lygčiu, takumo sąlygu ir geometriniu lygčiu diskretinès išraiškos baigtiniam elementui (10)-(12) ir konstrukcijos diskretiniam modeliui (14)-(17). Takumo sąlygos diskretizuojamos panaudojant klasikinius kolokaciju metodus: kolokacijų taške, kolokaciju srityje (elemente) ir Bubnovo-Galiorkino [11,12]. Diskretinio modelio pusiausvyros lygtys (14) sudaromos naudojant virtualiu poslinkiy principą, o geometrinés lygtys - virtualiy jègu principa.

Skirtingai nei kity autorių darbuose, takumo sąlygos ir geometrines lygtys sudaromos ne tik baigtinių elementy vidui, bet ir jų išoriniams paviršiams. Tai ir leidžia sudaryti nagrinejamų uždavinių diskretinius matematinius modelius (21)-(26), kuriuose jvertinami poslinkiu greičiu trūkiai ir energijos disipacijos greitis plastiniuose paviršiuse tarp baigtiniu elementu. Uždaviniu kinematines formuluotes matematiniai modeliai (22), (24), (26), (29) ir (32) sudaromi iš atitinkamy statinių formuluočiu Lagranžo daugikliu metodu. Taip pat pateikiami modifïkuoti uždaviniu matematiniai modeliai (27)-(29), kuriuose išeliminuotos 
pusiausvyros lygtys arba geometrinés lygtys pakeistos plastiniu deformaciju greixių darnos lygtimis (27), taip sumažinant lygčiu ir nežinomujų skaičiu.

Rèmo pavyzdžiu iliustruojama skaitmeniniu skaiciavimo rezultaty (ribinès apkrovos parametro) priklausomybè nuo lenkimo momenty aproksimavimo laipsnio ir nuo takumo sąlyg̨ diskretizacijos būdo. 1 lentelèje pateiktos rémo ribinès apkrovos parametro $F_{0}$ reikšmès ir ju procentinès paklaidos $\Delta F_{0}$ (palyginant su analitiniu sprendiniu $F_{0}=0,4662 M_{0}$ ), gautos pirmos ir antros eilès baigtiniams elementams (su atitinkamai tiesiniu ir paraboliniu lenkimo momenty pasiskirstymu), diskretizuojant rygelius $\mathfrak{i} 1,2,3$ ir 4 baigtinius elementus. Skaiciavimo rezultaty analize rodo, kad tiksliausi ir stabiliausi sprendiniai gaunami diskretizuojant takumo salygas Bubnovo-Galiorkino metodu. Diskretizuojant takumo sąlygas taškinès kolokacijos bei kolokacijos srityje būdu skaičiavimo rezultaty tikslumas priklauso ne tik nuo skaičiuojamojo tinklo tankio, bet ir nuo jo konfigūracijos daugiau ar mažiau sèkmingo pasirinkimo.

\section{THE PROBLEMS OF LIMIT LOAD ANALYSIS AND OPTIMIZATION USING EQUILIBRIUM FINITE ELEMENTS}

\section{S. Kalanta}

Su m ma ry

The equilibrium dual discrete mathematical models of the problems of limit load analysis and optimization are investigated in the article. These models are presented in terms of static and kinematic formulation using equilibrium finite elements. In these mathematical models the possible discontinuities of displacement velocities are evaluated and the velocity of energy dissipation is estimated not only within the volume of finite elements, but at the plastic surfaces between elements.

At first, on the basis of the energy principle of the maximum external power $[1,2]$ the general mathematical models (3) and (7) of static formulation of limit load analysis and optimization problems are created. In these models the yield conditions are controlled not only within the volume, but also at the surfaces of finite elements. The equilibrium finite elements and interpolation functions of strains (9) are used for discretization of these models. The constancy of external power is taken as the optimum criterion. The discrete expressions of fundamental relationships - equilibrium and geometric equations, yield conditions (10)-(12) for finite element and (14)-(17) for the discrete model of a body are developed. The discrete expressions of yield conditions are given using the classic collocation methods: collocation at the point, collocation at the sphere (element) and Bubnov-Galiorkin's collocation method $[11,12]$. The equilibrium equations of discrete structure are developed on the basis of virtual displacement principle while geometrical equations are derived using virtual force principle.

In contrast to the approach of other authors, yield conditions and geometrical equations are described not only within finite elements, but also at the surfaces between elements. That helps to design the dual discrete mathematical models of the problems (21)-(26), in which the discontinuities of displacement velocities and the velocity of energy dissipation in the place of those discontinuities are estimated. The mathematical models (22), (24), (26), (29) and (32) of kinematic problem formulation are developed from sensible static formulations by Lagrange's multiplier method. The modified mathematical models (27)-(29) are presented. In these models the equilibrium equations are eliminated or the geometrical equations are transformed into compatible equations of plastic stress velocities, in this way decreasing the number of equations and unknown values.

The dependence of the numerical results (limit load) of the frame on the approximation degree of bending moments, as well as on the discretization method of yield conditions are illustrated. In table 1 the values of limit loading parameter $F_{0}$ and their error of calculation $\Delta F_{0}$ (per cent, in comparison with the analytic solution $\left.F_{0}=0,4662 M_{0}\right)$ are presented. They are given for the first and second order finite elements with linear and parabolic distribution of bending moments using different discrete yield conditions and a different number of finite elements. The numerical result shows, that the discretization of yield conditions by Bubnov-Galiorkin's method gives the best accuracy and stable solutions. By discretizing yield conditions using the point's collocation and collocation at the element, the accurancy of numerical results depends not only on the number of elements, but also on a more or less successful choice of finite elements net.

Stanislovas KALANTA. Doctor, Associate Professor. Department of Structural Mechanics, Vilnius Gediminas Technical University, 11 Saulètekio Ave, 2054 Vilnius, Lithuania.

Doctor of technical sciences (structural mechanics), 1974. Probation: Leningrad Polytechnic Institute, Moscow Civil Engineering Institute, Kiev Civil Engineering Institute. Research interests: computational mechanics, finite element method, analysis and optimization of elasticplastic structures. 